\title{
Current Status of the Large-signal Code TESLA: Recent Development and New Applications
}

\author{
Igor A. Chernyavskiy, Alexander N. Vlasov, Simon J. Cooke, Baruch Levush \\ Naval Research Laboratory \\ Washington, DC 20375
}

\author{
Thomas M. Antonsen, Jr. \\ University of Maryland \\ College Park, MD 20742
}

\author{
Khanh T. Nguyen, Edward L. Wright \\ Beam Wave Research, Inc. \\ Bethesda, MD 20814
}

\begin{abstract}
TESLA is a $2.5 \mathrm{D}$ large-signal code, which is suitable for the modeling of single-beam and multiple-beam amplifiers based on linear propagating beams. Initially developed for the modeling of conventional, single-beam klystrons mainly, later on the code was extended and successfully validated to allow modeling of multiple-beam klystrons (MBKs), extended interaction klystrons (EIKs) and Inductive Output Tubes (IOTs). This work reports the current status of the code TESLA, including the latest advances in its development, validation and successful extension of its applicability.
\end{abstract}

Keywords: linear-beam amplifiers; large-signal code; klystron; multiple-beam.

\section{Introduction}

There is a big demand for high-power and high-efficiency RF power sources. Design and optimization of such devices requires use of efficient simulation tools. One such tool is the code TESLA, which currently is widely used in NRL and in a few large US tube companies. TESLA (Telegraphist's Equations Solution for Linear-beam Amplifiers) is a 2.5D large-signal code successfully applied to the modeling of single beam and multiple beam amplifiers having linear propagating beams. TESLA evolved from the code MAGY and was developed [1-3] as a result of collaboration of NRL, SAIC and the University of Maryland.

The current implementation of TESLA is based on the Fortran-95 language and takes advantage of the modern programming technique together with a highly efficient, dynamical use of the computer memory. On average, TESLA runs were found to be $\sim 2$ orders of magnitude faster than the typical runs of a fully 3D PIC code using the same CPU. Advanced performance of TESLA together with its user-friendly Python-based GUI and set of postprocessing tools makes the TESLA package very useful as a primary design tool. It has been verified, that the TESLA predictions are in good agreement with experimental data (see, for example [2-5]).

In this work we present the current status of the code TESLA, including the recent advances in its development, latest results of the TESLA modeling and its validation for extension of applicability of the code.

\section{TESLA modeling of the single-beam and multiple-beam ElKs}

The model initially developed in TESLA was perfectly suitable for modeling devices based on multiple-gap cavities. However, it is only recently that the code started to be seriously applied to such kind of configurations: successful modeling of the wide-band MBK-2 [4] was a first achievement in this direction. Then, more recently, we successfully have applied TESLA to the modeling of a Gband 6-cavity single-beam EIK and obtained very good agreement with an experiment [5].

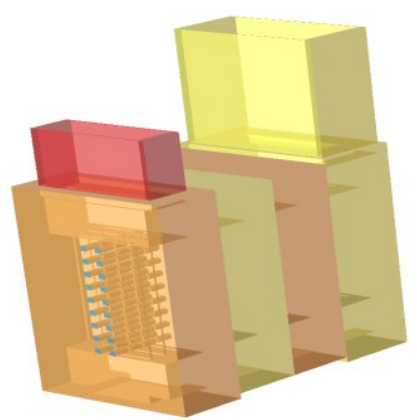

Figure 1: Schematic plot for the 4-cavity 18-beam EIK design (created by MAGIC-3D) [6].

Here we present the results of the modeling for the proposed 4-cavity 18-beam EIK [6], for which the initial design was performed using code MAGIC-3D (Fig.1). The typical run of the 3D code in this case takes tens of hours to complete, what makes it hard to use as an everyday design tool. On the other hand, the large-signal code TESLA was already successfully validated for multi-beam klystrons [3, 4] and single-beam EIK [5]. Therefore it was logical to assume that TESLA should be perfectly suitable for the modeling of the multiple-beam EIK too.

The results of the TESLA simulations for the 4-cavity 18beam EIK (in approximation of identical multiple-beams) are presented on Fig. 2 and show that the code's predictions are in a very good agreement with the predictions of 
MAGIC-3D for all levels of input power. Such good agreement of TESLA with the predictions of the fully 3D code together with the fact that its run takes few minutes only, makes it very attractive as a fast and efficient design tool for such type of devices too.

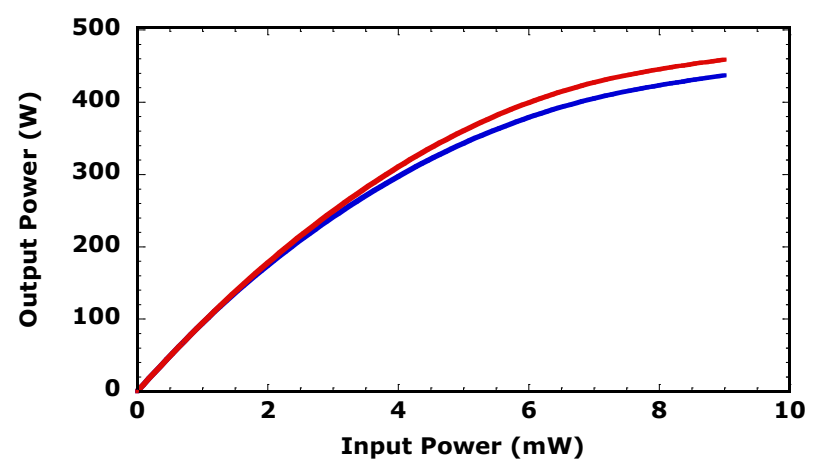

Figure 2: Comparison between the drive curves of the multiple-beam EIK, computed by the codes MAGIC (lower curve) and TESLA (upper).

\section{TESLA modeling of the single-beam and multiple-beam IOTs}

The capability of TESLA to utilize a pre-bunched beam was checked by applying it to the modeling of IOT type devices, based on the use of density modulated beams. Already the first tests, while using a simplified model for the density modulated beam, gave us very promising results (Fig.3). As a next step, in the code was added the capability to work with an arbitrary electron current or particle distribution, imported from a guncode. Together with recently developed depopulation algorithms for efficient particle importing into TESLA, the code became especially useful as a tool for a more realistic and direct modeling of IOTs in a close integration with the guncode MICHELLE [7].

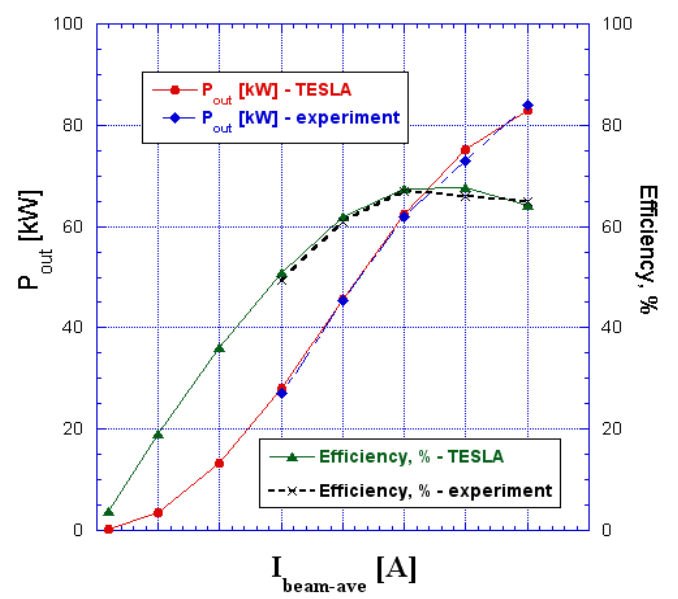

Figure 3: Comparison between the measured and predicted by TESLA output power and efficiency of the single-beam IOT (parameters and measured data courtesy of CPI, Palo Alto, CA).
Existing in TESLA accurate treatment for slow and reflected particles makes it especially suitable for the modeling of highly efficient IOTs (Fig. 4). Also, TESLA's multiple-beam algorithm makes the code fully applicable to the modeling of the multiple-beam IOTs too.

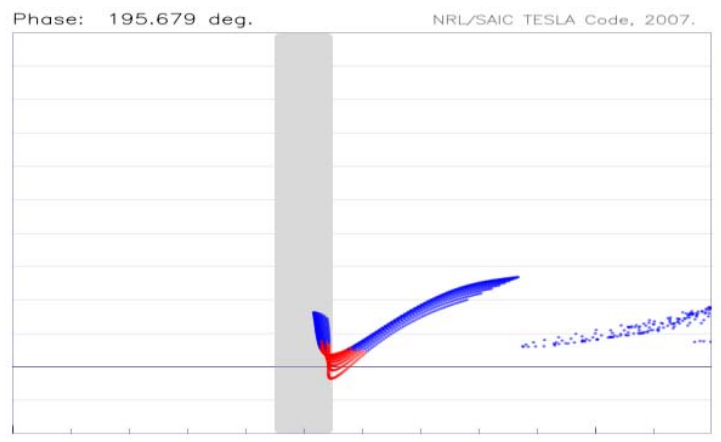

Figure 4: Snapshot of the simulated by TESLA phase-space of the electron bunch, shown here at the moment of its strong interaction with the RF fields near the gap of output cavity of a single-beam IOT.

\section{Acknowledgements}

This work was supported by the U.S. Office of Naval Research.

\section{References}

1. Vlasov, A.N. et. al., "Simulation of microwave devices with external cavities using MAGY", IEEE TPS, vol. 30, no. 3, pp.1277-1291, June 2002.

2. Cooke, S.J. et. al., "Validation of the Large-Signal Klystron Simulation Code TESLA," IEEE TPS, vol. 32, no. 3, pp.1136-1146, June 2004.

3. Chernyavskiy, I.A. et. al., "Parallel Simulation of Independent Beam-Tunnels in Multiple Beam Klystrons Using TESLA", IEEE TPS, vol. 36, no. 3, pp.670-681, June 2008.

4. Abe, D.K. et. al., "Experimental Study and Analysis of an S-band Multiple-Beam Klystron with 6\% Bandwidth”, IEEE TED, vol. 56, no.5, pp.846-854, May 2009.

5. Chernyavskiy, I.A. et. al., "Validation Study of the TESLA Model for Extended Interaction Klystron", 2011 IEEE Int'l Vacuum Electronics Conf., Bangalore, India, February 21-24, 2011, pp. 405-406.

6. Nguyen, K.T. et. al., "Multiple-beam Amplifiers", Presented on Conference ICOPs 2011, Illinois, Chicago, June 26-30, 2011.

7. Wright, E.L., et.al., "Multiple-Beam Inductive Output Tube Designs for High Average-Power Accelerator Applications," Presented on PAC09 Conference, Vancouver, Canada, May 4-8, 2009. 